vol. 29 - $n^{\circ} 4$ | 2013

Numéro ouvert

\title{
Processus et acteurs d'une vision sécuritaire des migrations : le cas du Canada
}

Process and Agents of the Securitization of Migration: The Case of Canada Proceso y actores de una visión de seguridad de las migraciones: el caso de Canadá

\section{Philippe Bourbeau}

\section{(2) OpenEdition}

\section{Journals}

\section{Édition électronique}

URL : https://journals.openedition.org/remi/6617

DOI : $10.4000 /$ remi.6617

ISSN : $1777-5418$

Éditeur

Université de Poitiers

Édition imprimée

Date de publication : 1 décembre 2013

Pagination : 21-41

ISBN : 979-10-90426-10-8

ISSN : 0765-0752

Référence électronique

Philippe Bourbeau, «Processus et acteurs d'une vision sécuritaire des migrations : le cas du Canada », Revue européenne des migrations internationales [En ligne], vol. 29 - n4 | 2013, mis en ligne le 01 décembre 2016, consulté le 14 avril 2022. URL : http://journals.openedition.org/remi/6617 ; DOI : https://doi.org/10.4000/remi.6617 


\section{Processus et acteurs d'une vision sécuritaire des migrations : le cas du Canada}

\section{Philippe Bourbeau ${ }^{1}$}

\section{Introduction}

Le phénomène migratoire compte parmi les grandes questions mondiales qui jalonneront le XXle siècle, tant il est vrai que les personnes en mouvement de par le monde sont aujourd'hui plus nombreuses qu'elles ne l'ont jamais été. On estime que plus de 200 millions de personnes se trouvent aujourd'hui hors de leur pays de naissance, ce qui représente environ $3 \%$ de la population mondiale. En d'autres termes, une personne sur trente-cinq dans le monde est un migrant (OIM, 2011).

Qui plus est, le trafic de migrants rivalise avec le trafic de stupéfiants en tant que source majeure de revenus de la criminalité organisée. La traite des êtres humains constitue un problème d'envergure mondiale. La migration "irrégulière " s'est hissée au premier rang des préoccupations des pays développés, à telle enseigne qu'elle figure maintenant en tête de l'agenda politique des pays du G8.

La perception des migrations internationales a aussi significativement changé depuis la fin de la guerre froide. Auparavant largement traitées sous un angle économique, les migrations internationales sont depuis quelques années une préoccupation de sécurité de la majorité des pays membres de l'OCDE. Des accords bilatéraux et multilatéraux ont été ratifiés, des institutions et agences ont été créées, des accords d'extradition ont été négociés et entérinés, et des conventions et des protocoles ont été ratifiés avec au cœur de tous ces instruments de la gouvernance le lien entre migration et sécurité. On observe par exemple une augmentation drastique de la détention des migrants dans les pays de l'OCDE depuis les années 1990. En France, le nombre d'immigrants placés en détention (centres de rétention administrative et zones d'attente) est passé de 23000 à 68900 entre 1999 et 2010 (ClCl, 2012 ; Collectif, 2012 ; La Cimade, 2012). Au Canada, le nombre d'immigrants légaux ou illégaux placés en détention

1 Maître de conférences au département de Science politique et chercheur associé à la Chaire Tocqueville en politique de sécurité de I'Université de Namur, 8 Rempart de la Vierge, Namur, Belgique ; philippe.bourbeau@unamur.be 
a plus que doublé entre 1996 et 2008 (Auditor General of Canada, 2008 ; CIC, 1996). On observe également un durcissement du contrôle à la frontière : aux États-Unis, à la fin des années 1990 (c'est-à-dire avant les attentats terroristes de 2001), I'agence responsable de l'immigration avait plus d'employés avec un port d'arme que n'importe quelle autre agence fédérale (US Department of Justice, 2000).

Pour décrire ce phénomène, les chercheurs parlent de la sécuritisation des migrations et de son processus, c'est-à-dire le processus par lequel les migrations sont intégrées dans un système de sécurité qui met l'emphase sur la défense et le contrôle (Bourbeau, 2011 ; Buzan et al., 1998 ; Huysmans, 2006). De plus, des indicateurs ont été développés afin de mieux cerner le phénomène de la sécuritisation des migrations internationales. Certains ont mis en avant deux catégories d'indicateurs. Dans un premier temps, les indicateurs de type " institutionnel " mettent en lumière la mention (ou non) des migrations internationales dans les énoncés de politique officielle en matière de politique étrangère, de sécurité et de défense, et dans les principaux textes de loi. Dans un deuxième temps, les indicateurs de type " pratiques sécuritaires " soulignent la présence (ou non) de pratiques sécuritaires appliquées au domaine des migrations internationales. Dans quelle mesure les pratiques d'interdiction sont-elles appliquées (Dench et Crépeau, 2003 ; Pratt, 2005 ; Rodier, 2006) et dans quelle mesure les pratiques de détention des migrants sont-elles répandues ? Sur la base de ces indicateurs, Bourbeau (2011) a pu mieux décrire la sécuritisation des migrations internationales dans son analyse comparée du Canada et de la France tout en soulignant d'importantes différences entre les deux pays, notamment en ce qui concerne les pratiques de détention des immigrants. En effet, entre 2000 et 2010 plus de 11000 individus en moyenne par année ont été placés en détention au Canada, alors que pour la même période en France le nombre s'élève à plus de 45000 individus.

De toute évidence, il existe depuis longtemps de nombreux mécanismes nationaux et internationaux de contrôle des flux migratoires. De même, l'idée que certains individus constituent une menace pour la sécurité n'est pas nouvelle. Ce qui est nouveau et hautement significatif est pour certains (a) l'idée que les migrations, au sens collectif du terme, représentent une question de sécurité nationale et/ou sociétale ; (b) le haut niveau de danger "sécuritaire " souvent associé aux migrations; et enfin (c) l'élaboration par de nombreux pays d'un vaste ensemble de pratiques sécuritaires visant directement la " menace " migratoire.

L'étude de la sécuritisation des migrations est un des axes de recherche des plus dynamiques en sciences sociales (Adamson, 2006 ; Bigo, 1998a et 1998b ; Bigo et Tsoukala, 2008 ; Bourbeau, 2011 et 2013a ; Ceyhan et Tsoukala, 2002 ; Clochard, 2010 ; Huysmans, 2006 ; Van Munster, 2009). Or, si la littérature théorique sur le processus de sécuritisation est bien développée, la base empirique demeure incomplète et limitée, particulièrement l'analyse de contenu de discours réalisée de façon systématique et sur une période de plusieurs années.

Deux objectifs guident cet article. D'une part, nous cherchons à décrire et à analyser, sur la base d'une étude comparative des discours de plusieurs 
agents politiques au Canada entre 1989 et 2011, le rôle de nombreux agents dits " sécurisateur " ${ }^{2}$ dans le processus de sécuritisation des migrations. Plusieurs questions guident notre analyse : ces agents ont-ils cherché à présenter les migrations internationales comme étant une menace sécuritaire à l'État, à la société canadienne, à l'identité collective, ou à la cohésion sociale du pays ? Quelles ont été les raisons justifiant les tentatives de sécuritisation invoquées par ces agents ? Le "Canada " a-t-il " parlé " d'une seule voix dans ce dossier ou une analyse détaillée révèle des positions contradictoires et une fragmentation de la position canadienne ? D'autre part, nous tenterons de dégager, en conclusion et sur la base de notre analyse, des hypothèses de travail pour les études sur la sécuritisation des migrations.

En ce sens, notre approche est d'abord orientée vers une description des mécanismes sociaux propres à la sécuritisation des migrations plutôt que vers une explication de la sécuritisation (Berthomière et Hily, 2006). Dans le même ordre d'idée, nous ne cherchons pas à identifier les " auteurs " uniques du processus social qu'est la sécuritisation des migrations. Le processus est complexe et il ne saurait être réduit au rôle d'un seul agent. En ce sens, nous concordons avec Hannah Arendt (1961 : 207-208) en postulant que :

" Les histoires, résultats de l'action et de la parole, révèlent un agent, mais cet agent n'est pas l'auteur, n'est pas producteur... En toute série d'événements qui ensemble forment une histoire pourvue d'une signification unique, nous pouvons tout au plus isoler l'agent qui a mis le processus en mouvement... nous ne pouvons jamais le désigner sans équivoque comme l'auteur des résultats éventuels de cette histoire ".

Notre argumentation comprend deux parties. Nous identifierons tout d'abord le cadre d'analyse utilisé afin de rendre compte du rôle de certains agents politiques dans le processus de sécuritisation des migrations. Nous présenterons par la suite les résultats de notre analyse de contenu des discours du Premier ministre, du ministre des Affaires étrangères et du ministre de la Citoyenneté et de I'Immigration du Canada entre 1989 et 2011. Enfin les pistes de réflexions créées par notre étude seront abordées en conclusion.

\section{Le processus de sécuritisation : actes de langage et facteurs contextuels}

La nature des mécanismes de la sécuritisation des migrations a suscité d'intenses débats et plusieurs arguments et modèles d'explication ont été avancés ces dernières années. Certains chercheurs ont analysé l'influence des diasporas sur les politiques étrangères et de sécurité d'un pays ou sur la mondialisation (Bourbeau, 2001 et 2002 ; Loch et Barou, 2012 ; Loescher, 1992 ; Newman et Van Selm, 2003 ; Shain et Barth, 2003). D'autres ont mis en lumière les effets de

\footnotetext{
2 Nous introduisons ici ce néologisme pour désigner les agents qui formulent des tentatives de sécuritisation, c'est-à-dire un acte de langage par lequel un agent avance l'argument que les migrations internationales sont une menace existentielle et un danger pour la sécurité d'un objet de référence (que ce soit l'État, la société, l'identité collective, etc.). Tous les agents sécurisateurs n'ont évidemment pas le même pouvoir social. De même certains agents sont dits " institutionnels " de par la position sociale qu'ils occupent alors que d'autres sont dits "émergents ", voir Bourbeau (2011), Buzan, Waever et al. (1998) et Waever (1995).
} 
la sécuritisation des migrations sur les immigrants eux-mêmes (Agier, 2002 ; Barutciski, 2001 ; Caloz-Tschopp, 2004 ; Crépeau et Nakache, 2006 ; Freedman, 2004 ; Kobelinsky et Makaremi, 2008 ; Le Bail, 2008 ; Martiniello et Simon, 2005), parfois via les questions d'intégration, de citoyenneté et d'idéologies d'aménagement de la diversité (Bloemraad, 2006 ; Brubaker, 1992 ; Geddes, 2012 ; Guiraudon, 2000 ; Helly, 2009 ; Joppke et Morawska, 2003 ; Noiriel, 2008 ; Rea et Tripier, 2008 ; Tribalat, 2012 ; Winter, 2011 ; Wihtol de Wenden, 2010). D'autres encore ont étudié l'évolution de la capacité de contrôle des flux migratoires par les États, le plus souvent de façon comparative (Andreas, 2000 ; Cornelius et al., 2004 ; Guiraudon, 2010 ; Guiraudon et Joppke, 2001 ; Hollifield, 2004 ; Pellerin et Gabriel, 2008).

Certains chercheurs ont présenté une vision anarchique, alarmiste et déterministe de l'influence des flux migratoires sur l'ordre international. Selon Kaplan, les pays occidentaux devraient craindre "l'anarchie à venir " créée par les migrations de masse (Kaplan, 1994). Pour Huntington, l'immigration hispanique aux États-Unis constitue une menace sécuritaire potentielle à l'identité culturelle et politique du pays (Huntington, 2004). D'autres encore voient le monde divisé en deux camps : d'un côté les riches, de l'autre les pauvres. Or si les "riches " ne se protègent pas, les "pauvres " vont tôt ou tard les envahir (Connelly et Kennedy, 1994). Certains commentateurs vont plus loin en postulant que par définition les migrations sont un problème de sécurité puisque les migrations modifient les sociétés d'accueil et donc mettent en péril l'équilibre sociétal et identitaire d'une société (Miller, 1998). Enfin, certains internationalistes estiment que les migrations sont une question de sécurité pour les pays occidentaux parce qu'elles ont la capacité de provoquer des conflits internationaux qui pourraient avoir des conséquences sur l'intérêt national et la sécurité des pays occidentaux (Weiner, 1993 et 1995).

Plusieurs chercheurs ont cependant fait remarquer que ce type d'approche posait problème à de nombreux niveaux (Badie et Wihtol de Wenden, 1994 ; Doty, 1998 ; Guild et Van Selm, 2005 ;Tirman, 2004). En effet, I'argument selon lequel les migrations constituent, structurellement et de façon déterministe, une question de sécurité représente un fondement théorique plus qu'incertain sur la base duquel construire un cadre d'analyse du processus de sécuritisation. De plus, plusieurs études récentes viennent également contredire l'argument que les migrations soient vues sous un angle de sécurité par les pays occidentaux parce qu'elles provoquent des conflits internationaux mettant en jeu les intérêts nationaux des pays occidentaux (Bourbeau, 2011 ; Curley et Wong, 2008 ; Pratt, 2005).

Influencés par l'approche néo-libérale institutionnelle, certains auteurs avancent l'argument selon lequel les migrations internationales sont vues sous un angle sécuritaire parce qu'elles représentent une menace structurelle géopolitique. Concevant l'État comme un acteur rationnel, unitaire et autonome, ces chercheurs tentent de démontrer que les différents intérêts domestiques d'une société sont amalgamés et ensuite traduits par "l'intérêt national " au nom duquel l'État agit. En ce sens, le processus de sécuritisation des migrations est le même à travers les études de cas et est d'abord et avant tout une réponse à une menace structurelle extérieure (Hollifield, 2000 ; Rudolph, 2006). Cependant, une telle approche n'offre pas la distance nécessaire pour étudier 
pleinement cette évolution, et ce pour deux raisons. D'une part, traiter l'État comme un agent rationnel et unitaire limite plus que n'éclaire dans le présent contexte car, comme nous le verrons dans la prochaine section, "la " position du Canada face aux migrations n'a pas été dans les deux dernières décennies une position unitaire. D'autre part, une telle perspective ne permet pas de dégager les éléments de ruptures et de continuités dans le processus et représente un exemple de ce qu'Andreas Wimmer et Nina Glick Schiller (2003) ont appelé le " nationalisme méthodologique " - c'est-à-dire, la " naturalisation de l'Étatnation par les sciences sociales ".

Une des approches les plus influentes dans le monde de la recherche francophone sur la sécuritisation est la thèse de la gouvernementalité par l'inquiétude avancée par Didier Bigo et ses collaborateurs (Bigo, 1998b et 2005 ; Bigo et Guild, 2003 ; Bigo et Tsoukala, 2008 ; Ceyhan et Tsoukala, 2002). Inspiré par les travaux de Michel Foucault et de Pierre Bourdieu, Bigo soutient que la sécurité n'est pas une question simplement de survie et ne fait pas appel à un sentiment d'urgence ou à des pratiques exceptionnelles. Le processus de sécuritisation des migrations serait $d^{\prime}$ 'abord et avant tout fonction des pratiques routinières des professionnels de la sécurité (c'est-à-dire la police et les agents de douanes) qui créent et reproduisent de façon diffuse un système de peur et d'inquiétude ${ }^{3}$.

La thèse de l'inquiétude est souvent contrastée avec la thèse des actes de langage sécuritaire développée par Barry Buzan et Ole Wæver (Buzan et Wæver, 2003 ; Buzan et al., 1998 ; Wæver, 1995). Inspirés à la fois des travaux de Carl Schmitt et de John Austin, Buzan et Wæver postulent que la sécurité répond à une menace existentielle, qu'elle suit une logique d'exception et que le pouvoir discursif est central dans la construction sociale de ce qu'est une question de sécurité.

En effet, les philosophes ont longtemps pensé qu'il n'y avait que des énoncés de constat, et donc des énoncés vrais ou faux. Austin (1991) a montré l'importance d'admettre l'existence des énoncés performatifs, c'est-à-dire une énonciation qui ne fait pas que décrire mais qui " fait " elle-même quelque chose. L'exemple typique est la phrase " je vous déclare mari et femme ". II y a plus dans l'énonciation de cette expression que la description d'une réalité ; dire cette phrase, c'est accomplir un acte transformateur. Dans ce cas-ci, la phrase fait changer de statut les personnes concernées.

Or, dans l'étude de la sécuritisation, un acte de langage présente les migrations comme une menace sécuritaire existentielle à l'identité collective, à la société ou à l'État. Une tentative de sécuritisation, ou un acte de langage de sécurité, n'est pas automatiquement efficace, c'est-à-dire qu'elle ne crée pas nécessairement l'effet escompté. De même, ce ne sont pas tous les agents qui sont susceptibles de formuler un acte de langage efficace. Un agent " sécurisateur " doit posséder un pouvoir et une reconnaissance sociale particulière pour énoncer une tentative de sécuritisation qui soit prise en considération. 
D'une part, et faisant écho aux critiques que Bourdieu (1982) a adressées à Austin sur la nécessité de prendre en compte dans l'analyse des énoncés performatifs les rapports sociaux intrinsèques à l'espace social, il est important de reconnaitre que le pouvoir social d'un agent peut être institutionnel. Ainsi, les positions sociales peuvent avantager certains agents dans la formulation - et la prise en considération par les autres agents - d'actes de langage présentant les migrations internationales comme une question de sécurité. Les agents, dits institutionnels, sont susceptibles de posséder un pouvoir social qui renforce à la fois leur légitimité à parler en termes sécuritaires et la prise en compte par les autres agents de leurs actes de langage. De toute évidence, les agents qui occupent des fonctions politiques sont les exemples classiques d'agents institutionnels: les premiers ministres et ministres fédéraux et provinciaux. Cependant, dans le contexte de la sécuritisation des migrations il ne faut pas oublier les agents médiatiques, les maires des grandes villes, les membres de groupe de lobby, etc. En somme, la catégorie d'agents désireux et aptes à énoncer des actes de langage de sécurité doit rester théoriquement et empiriquement ouverte; et les outils analytiques nous permettant de mieux comprendre le phénomène de la sécuritisation des migrations gagnent à demeurer également ouverts.

D'autre part, et faisant écho aux critiques que Judith Butler (1997 et 1999) a formulé envers Bourdieu sur l'importance d'accepter l'idée que les positions sociales sont elles-mêmes le résultat d'une construction à travers le processus de performativité et qu'il existe une différence entre " être autorisé à parler " et " parler avec autorité " puisqu'il est possible de "parler avec autorité sans être autorisé ", il est également important de reconnaître que le pouvoir social d'un agent peut être émergent. En effet, il est possible qu'un agent ait peu de pouvoir social au début du processus mais que son pouvoir augmente précisément grâce aux questions qu'il soulève.

Non seulement il est essentiel d'étudier les relations entre agents mais il est également fondamental $d$ 'analyser les actes de langages en regard du contexte dans lequel ils ont été formulés. Les tentatives de sécuritisation naissent de l'environnement dans lequel elles sont énoncées et sont contraintes par ce même environnement. En fait, le processus de sécuritisation fait appel à la constitution mutuelle de l'agent et du contexte. Concevoir la relation entre les agents et les facteurs contextuels comme étant mutuellement constituée permet non seulement d'inclure les notions de feed-back et de multi-directionalité dans l'étude des mécanismes sociaux liés à la sécuritisation des migrations, mais permet également une meilleure compréhension des dynamiques entre les agents et les différents contextes propres au processus de sécuritisation des migrations.

Les agents n'évoluent pas dans un vacuum social. Les agents " sécurisateurs " ne peuvent s'exclure de l'environnement contextuel dans lequel ils évoluent. Certes, les agents possèdent un degré considérable d'autonomie mais leur capacité à reproduire ou à transformer le champ sécuritaire n'est pas sans contraintes et sans limites. De même, la signification et la construction des structures sociales sont dépendantes de l'interprétation des agents. Les facteurs contextuels n'existent pas de façon objective dans I'espace social en attente d'exercer leurs influences. Les divers contextes dans lesquels les agents opèrent ne peuvent imposer la sécuritisation des migrations d'eux-mêmes. Un 
facteur contextuel doit être interprété comme ayant une signification en termes de sécurité pour que ce facteur possède un impact sécuritaire. Les structures sociales possèdent le pouvoir de légitimer et d'autoriser, d'inclure et d'exclure, et ainsi d'exercer un pouvoir considérable sur les agents, notamment en accréditant de l'autorité à un agent ou à des agents particuliers. Les positions structurales génèrent des positions et des capacités sociales inégales.

II nous est impossible dans un article comme celui-ci d'analyser toutes les dimensions de ce sujet. Le choix de restreindre notre champ aux discours d'agents politiques n'est pas un choix théorique mais représente plutôt une volonté de produire une analyse systématique, rigoureuse et en profondeur d'un élément du cadre d'analyse permettant l'étude du processus de sécuritisation des migrations ${ }^{4}$.

\section{Agents, sécuritisation et migration : le cas du Canada}

Afin de bien cerner le rôle de certains agents politiques dans le processus de sécuritisation, nous avons archivé puis analysé l'ensemble des discours prononcés par le Premier ministre, le ministre des Affaires étrangères et le ministre de la Citoyenneté et de l'Immigration du Canada entre 1989 et $2011^{5}$. Ce corpus de recherche représente plus de 1700 discours. Pour chaque discours, nous avons cherché à comprendre comment les migrations étaient perçues, discutées et représentées. De quoi parlaient ces agents lorsqu'ils discutaient des migrations : était-ce en référence aux avantages du multiculturalisme, aux difficultés des immigrants à s'intégrer sur le marché du travail, à l'apport considérable des immigrants à la société canadienne, au "problème " que représente l'immigration pour la société d'accueil, etc. Nous avons par la suite isolé les tentatives de sécuritisation : c'est-à-dire ces agents ont-ils déclaré que les migrations internationales sont une menace à la sécurité du Canada et/ou à l'identité collective canadienne ? Nous avons tenté de présenter les résultats de façon chronologique.

Barbara McDougall, ministre des Affaires étrangères de 1991 à 1993, est l'un des premiers agents politiques ayant formulé des tentatives de sécuritisation au cours des années relatives à l'étude. Dans un discours en décembre 1991, McDougall déclare qu'en adoptant un concept plus large de la sécurité, le Canada sera plus " agressif et actif " dans la lutte contre les menaces transnationales à la sécurité telles que le trafic de drogue, le terrorisme et les migrations "irrégulières " (McDougall, 1991). À la rencontre du Conseil des ministres de la Conférence sur la sécurité et la coopération en Europe, McDougall renchérit et affirme qu'adopter une approche coopérative de la sécurité entre les États membres permettrait de "réduire la menace des migrations internationales de

\footnotetext{
4 Voir Bourbeau (2011) pour un traitement plus exhaustif.

5 De toute évidence, un nombre considérable d'agents politiques autres que ceux sélectionnés auraient pu être étudiés. En concentrant notre analyse sur les agents politiques en position de pouvoir institutionnel nous avons voulu à la fois limiter le corpus de recherche (déjà imposant) et avancer les premiers éléments nécessaires à un projet de recherche plus large visant à détailler et décrire le rôle de nombreux autres agents politiques dans le processus de sécuritisation au Canada.
} 
masse " (McDougall, 1992a). La nécessité d'adopter une vision sécuritaire dans la gestion des mouvements migratoires de masse est réaffirmée à plusieurs reprises au début des années 1990. Lors de l'ouverture officielle de I'Institut canadien du Service extérieur à Ottawa, McDougall avance l'idée que " les défis de nos services étrangers sont maintenant plus complexes et plus diversifiés qu'avant. Les migrations de masse ont changé pour toujours le travail de nos agents d'immigration - un travail qui est essentiel pour assurer la sécurité de tous les Canadiens " (McDougall, 1992b).

Alors que le Premier ministre Brian Mulroney (1984-1993) ne fera pas écho aux arguments de sa ministre des Affaires étrangères, son successeur à la tête du Parti progressiste-conservateur et du même coup comme Première ministre par intérim, Kim Campbell (Juin 1993-Novembre 1993), pose dans I'un de ses premiers discours comme Première ministre les termes du débat. Par la création d'un nouveau ministère de la Sécurité publique consolidant les responsabilités dans les domaines policiers et du traitement des demandes d'application d'immigration, Campbell (1993) entend assurer que la société canadienne ne soit pas exposée à d'importants risques sécuritaires découlant de la menace migratoire.

Avec l'arrivée au pouvoir du Parti libéral du Canada, sous la gouvernance de Jean Chrétien (1993-2003), le ministère de la Sécurité publique et le narratif entourant le lien migration-sécurité disparaissent de l'espace publique pour quelques années. Entre 1989 et 2001 (et mise à part la " parenthèse " Campbell de 1993), aucune tentative de sécuritisation des migrations ne sera prononcée par le Premier ministre canadien.

Ce ne sont pas les occasions qui manquent par contre. En effet, à l'été 1999, quatre bateaux transportant des migrants d'origine chinoise échouent au large de la Colombie-Britannique, et le débat sur les lois et les politiques relatives à l'immigration et au statut de réfugié prend alors une ampleur et une intensité renouvelées. Les quelque 600 personnes d'origine chinoise avec la présence d'adolescents, sont arrivées dans des conditions exécrables, à bord de navires qui prenaient l'eau ; aucun n'avait de papiers et la plupart ont demandé le statut de réfugié. La couverture médiatique fut imposante et plusieurs études ont démontré le rôle joué par les médias dans la sécuritisation des migrations suite à cet évènement (Bradimore et Bauder, 2011 ; Mountz, 2004 et 2010 ; OxmanMartinez, et al., 2005 ; Pratt, 2005). Ce qui est maintenant communément appelé "l'été chinois de 1999 " aurait pu facilement servir de courroie au Premier ministre pour populariser l'idée que cette migration de masse est un exemple frappant des nouvelles dynamiques sécuritaires en cette fin de XXe siècle auquel le Canada doit maintenant faire face. Cependant, le Premier ministre n'a prononcé aucune tentative de sécuritisation de l'immigration.

Le ministre des Affaires étrangères (Llyold Axworthy, 1996-2000) et la ministre de la Citoyenneté et de I'Immigration (Elinor Caplan, 1999-2001) interprèteront de façon bien différente "l'été chinois de 1999 ". D'une part, Axworthy verra dans cet évènement une illustration de la doctrine de sécurité humaine qu'il défend depuis plusieurs années. C'est-à-dire, que l'arrivée par bateau de réfugiés "démontre aux Canadiens I'horrible réalité d'une autre menace à la sécurité humaine : le trafic et la traite d'êtres humains " (Axworthy, 1999). Ainsi l'arrivée par bateau de réfugiés ne doit pas être comprise en termes de menace 
à la sécurité du Canada (ou de la société canadienne) mais bien en termes de menace à la sécurité des réfugiés et immigrants forcés de se déplacer dans des conditions qualifiées d'inhumaines. Cette position sera maintenue et développée à plusieurs reprises, comme l'illustre ce passage :

"Des millions d'individus vulnérables sont forcés de fuir leurs maisons, se présentent à des frontières qui sont ouvertes une minute puis fermées aussitôt, sont poussés à se cacher, sont séparés de leurs familles, perdent leurs identités, sont abusés sexuellement et cruellement tués. La nécessité de combattre ces menaces à la sécurité humaine est à la base de la politique étrangère du Canada " (Axworthy, 2000).

D'autre part, Caplan traduira " l'été chinois de 1999 " avec un tout autre angle sécuritaire. Dès I'un de ses premiers discours suivant la crise politique, Caplan soutien qu'une politique accrue de détention des réfugiés arrivés par bateau est nécessaire pour assurer la sécurité du Canada.

" Nous savons que si un processus accéléré des demandes d'immigration est une partie de la solution, une politique de mise en détention de ces individus l'est également. Nous avons déjà annoncé des propositions afin d'augmenter notre capacité à placer en détention un individu si cet individu ne possède pas de documentation valide et s'il ne coopère pas avec les autorités" (Caplan, 1999b).

Cette position est réaffirmée à plusieurs reprises dans les mois qui suivent I'arrivée des bateaux de réfugiés (Caplan, 1999a, 1999c, 2000a et 2000b). Parfois, le groupe cible est clairement identifié, "les étrangers condamnés pour des crimes graves, les criminels de guerre, les terroristes, ceux qui représentent un risque pour la sécurité nationale. Je les veux hors d'ici. Ils sont strictement inadmissibles et ne sont pas les bienvenus " (Caplan, 2000d). Parfois, la cible est plus diffuse, " comment maintenir un juste équilibre entre nos objectifs humanitaires concernant les nouveaux arrivants et la nécessité d'assurer la sécurité du Canada face au flux migratoire mondial ? "(Caplan, 2000c).

Le successeur d'Axworthy comme ministre des Affaires étrangères, John Manley (2000-2002), poursuivra ce travail de sécuritisation des migrations dès son entrée en fonction mais en qualifiant davantage quel type d'immigration serait considérée comme une question de sécurité. "Nous faisons face à de nouvelles et complexes menaces sécuritaires ", déclare Manley en 2000, " et ces menaces incluent les migrations illégales, le crime et le terrorisme " (Manley, 2000).

Tandis que I'arrivée de quatre bateaux à l'été 1999 initie une lecture sécuritaire des migrations chez la ministre en charge de l'immigration, les attentats terroristes du 11 septembre 2001 viendront durcir la position officielle. Dans l'un de ses premiers discours suivant les attentats, Caplan (2001b) décide d'envoyer un message sans équivoque :

"Les Canadiens comptent sur nous pour assurer la sécurité de notre société. Je suis fière de dire que c'est une loi [Loi sur l'immigration et la protection des réfugiés] très dure pour ceux qui sont une menace à la sécurité du Canada... Nous nous attaquerons aux criminels et aux menaces à la sécurité ". 
Au cours des mois suivants, les attentats du 11 septembre 2001, la ministre ne cessera de pousser une lecture sécuritaire des migrations internationales. "Dans mon propre portefeuille de responsabilité - l'immigration - nous avons agi rapidement et fermement dans le but de faire face aux menaces à la sécurité et la protection des Canadiens " (Caplan, 2001a).

Ces thèmes seront également repris par le Premier ministre. Dans son discours lors du débat spécial de la Chambre des communes en réponse aux attentats terroristes, le Premier ministre Jean Chrétien n'a pas hésité à établir un lien fort entre migration et sécurité. Cependant, il s'empresse de faire remarquer que le Canada ne sombrera pas dans la tentation de créer un cordon sécuritaire autour du pays et que son gouvernement ne versera pas " dans l'espoir - inutile et ultimement contreproductif - de faire du Canada une forteresse contre le monde extérieur " (Chrétien, 2001).

Malgré ces remarques, I'arrivée de Denis Coderre (2002-2003) comme nouveau ministre en charge du dossier de l'immigration durcira considérablement le ton. Rappelant que son ministère opère dans un environnement hautement sécuritaire depuis le 11 septembre, il souligne que son ministère est extrêmement vigilant quant aux menaces à la sécurité nationale du Canada et que les Canadiens "peuvent être sûrs que nous faisons tout ce qui est en notre possible pour assurer la sécurité de la nation canadienne " (Coderre, 2003d). Se félicitant de l'adoption de mesures sécuritaires telles que la prise d'empreintes digitales systématique et la vérification que tous les demandeurs d'asile ne représentent pas un danger sécuritaire pour le Canada, Coderre formule de nombreuses tentatives de sécuritisation au cours de son mandat de près de deux ans. Selon Coderre (2003a et 2003c), non seulement le Canada vient d'entrer dans un " nouvel ordre mondial " mais ce nouvel ordre exige que tous les paliers du gouvernement adoptent de nouvelles approches afin d'assurer la sécurité du Canada. De plus, Coderre (2003c) met l'accent sur la surveillance et le renforcement des contrôles avec des dispositifs biométriques, notamment la création d'une carte d'identité nationale. II ne s'agit pas ici de céder aux pressions incessantes et répétées des États-Unis, prétend le ministre, mais bien d'une "question de sécurité internationale ". Selon Coderre, la nouvelle carte d'identité " empêchera le terrorisme " et gardera à distance les " menaces sécuritaires ». En octobre 2003, il ajoutera que :

"L'intégrité des documents est un moyen fondamental d'améliorer la sécurité nationale du Canada et des Canadiens dans un monde incertain. Elle est un élément important de notre stratégie afin d'assurer la sécurité des Canadiens " (Coderre, 2003b).

Lors des élections fédérales de 2006, le Parti libéral, en place depuis 1993, est chassé du pouvoir et c'est un gouvernement conservateur qui lui succède. D'abord minoritaire aux élections de 2006 et 2008, le Parti conservateur obtient une majorité parlementaire aux élections fédérales de 2011.

Les thèmes de danger sécuritaire et de menaces à la frontière du Canada demeureront au centre des préoccupations du ministre de la Citoyenneté et de I'Immigration. Monte Solberg (2006-2007), nouvellement nommé ministre en charge du dossier de l'immigration, estime que le Canada doit agir rapidement afin d'imposer des sanctions aux personnes entrées clandestinement au Canada, 
sans quoi " des centaines de milliers d'autres personnes tenteraient également $d^{\prime} y$ entrer clandestinement ". Les réponses du gouvernement canadien face au problème du système d'immigration, poursuit Solberg (2006b), doivent " garantir la sécurité des frontières et l'intégrité du système d'immigration ". Au fil des discours du ministre, " assurer la sécurité à nos frontières " est un thème qui revient à maintes reprises (Solberg, 2006a).

Jason Kenney (2008-2011), considéré comme l'un des ministres de la Citoyenneté et de l'Immigration les plus influents des dernières années, poursuit l'emphase du ministère sur la sécurité du Canada. Bien que le programme d'immigration du Canada garantisse le regroupement familial et la protection des personnes fuyant la persécution, et une politique attractive pour les migrants économiques, " nous devons protéger l'intégrité de notre programme si nous voulons défendre les Canadiens contre une gamme de risques liés à la santé et à la sécurité ", estime Kenney (2008).

De même, les thèmes d'immigration et de terrorisme sont également accolés et juxtaposés. Selon Kenney (2009), " le terrorisme est une réalité mondiale. C'est pourquoi l'immigration au Canada est réglementée par une loi exhaustive, en vertu de laquelle les personnes qui représentent une menace pour la sécurité du public, ou pour la sécurité nationale, sont interdites de territoire ". Plus récemment, le ministre a offert son analyse de la pièce maîtresse du système d'immigration canadien. Pour Kenney (2011), I'entrée en vigueur de la Loi sur l'immigration et la protection des réfugiés (2002) a eu pour effet de changer l'objectif de l'ancienne loi sur l'immigration, désormais il s'agit de " donner priorité à la sécurité ".

Tandis que les différents ministres de la Citoyenneté et de l'Immigration mettent l'accent sur l'intégrité du programme d'immigration et la nécessité de concevoir une politique sous un angle sécuritaire, le ministre des Affaires étrangères de 2008 à 2011, Lawrence Cannon, concentre ses remarques sur le thème des migrations clandestines et des menaces à la sécurité qu'elles représentent. Selon Cannon (2010), " les menaces à la sécurité transfrontalière et maritime posées par des terroristes, des pirates, des bandits et des trafiquants de drogue, d'armes et de migrants clandestins ne cessent d'augmenter ".

En somme, notre étude du rôle joué par différents agents politiques dans la sécuritisation des migrations au Canada entre 1989 et 2011 démontre la place centrale du ministre des Affaires étrangères dans l'initiation du processus de sécuritisation des migrations ; la peur des " migrations de masse " étant I'un des moteurs. II faut noter cependant que si le ministre des Affaires étrangères du Canada a été au cœur du processus de sécuritisation des migrations au début des années 1990 puis dans l'immédiat suivant les attentats terroristes du 11 septembre 2001, il a joué un rôle beaucoup plus efface dans les dernières années. À l'inverse, le ministre de la Citoyenneté et de l'Immigration fut largement absent du processus de sécuritisation des migrations dans les premières années couvertes par notre étude et n'a cessé de prendre de l'importance au sein du gouvernement depuis le choc exogène que fut " l'été chinois de 1999 ». Enfin, bien que la sécuritisation des migrations ait difficilement pu se faire sans l'engagement ou l'accord tacite du Premier ministre, notre analyse suggère que le Premier ministre a activement participé au débat public sur la 
sécuritisation des migrations seulement après les attentats terroristes du 11 septembre 2001, et ce pour une courte période de temps.

Notre étude révèle l'importance des actes de langages sécuritaires dans la construction sociale des migrations internationales comme un objet de sécurité au Canada. Depuis 1989, ces tentatives ont non seulement été nombreuses mais elles ont été formulées par plusieurs agents politiques issus de différents partis. Notre étude a également souligné l'importance du processus de sécuritisation qui s'opère après les évènements de l'été 1999. Le processus de sécuritisation fait appel à de nombreuses autres composantes sociales que les tentatives de sécuritisation de certains agents politiques décrites plus haut. En ce sens, l'objectif de cet article n'était pas d'identifier, de décrire et d'analyser l'ensemble des facteurs impliqués dans la sécuritisation des migrations internationales au Canada mais bien de réaliser quelques pas, aussi modestes soient-ils, vers une meilleure compréhension du rôle de certains agents dans ce processus sur plusieurs années.

\section{Conclusion}

La sécurité n'est pas une donnée objective répondant uniquement à des facteurs matériels ou à " la " réalité. L'identification d'une menace est une construction sociale. Le processus de sécuritisation des migrations requiert des mécanismes sociaux d'énonciation et de démonstration, la sécuritisation des migrations n'est ni automatique ni objective. Ainsi, le processus de sécuritisation répond à des logiques intersubjectives où interagissent les agents dits sécurisateurs et les facteurs contextuels à l'intérieur desquels opèrent ces agents. L'argument selon lequel les migrations représentent une menace à la sécurité d'un État, d'une société ou d'une identité collective doit être énoncé et défendu ; les migrations ne sont pas en soi une question de sécurité.

Or, nous avons tenté de décrire dans cet article le rôle joué par plusieurs agents politiques dans la sécuritisation des migrations au Canada entre 1989 et 2011 et les raisons invoquées par ces agents pour justifier les politiques mises en place. Cette recherche comble un vide dans la littérature notamment francophone. Elle ouvre également des pistes de recherches. Une première ligne de recherche concerne les outils développés pour décrire la sécuritisation des migrations. Les recherches portant sur les indicateurs et sur la variation intra et inter-cas de la sécuritisation des migrations ont certes débuté, mais beaucoup reste à faire surtout dans le cadre d'une analyse comparée (Bourbeau, 2011 ;Van Munster, 2009).

Un deuxième axe de recherche concerne la diversité des agents sécurisateurs. Dans cet article, nous avons limité notre analyse aux seuls agents politiques. Or, de nombreux autres agents participent à ces processus et leur rôle demeure sous-étudié, que l'on pense aux maires des grandes villes, aux agents médiatiques, à l'opinion publique ou encore aux entreprises privées de sécurité (Abrahamsen et Williams, 2011 ; Potvin, 2008 ; Rodier, 2012 ; Tsoukala, 2010).

Une troisième piste de recherche est celle de la contestation. Comme nous l'avons mentionné précédemment, le processus de sécuritisation n'évolue pas dans un vacuum social. Or, puisque la sécuritisation est socialement construite, 
nombre d'études ont aussi cherché à comprendre les mécanismes de contestation qui y sont liés, notamment la dé-sécuritisation et la résilience (voir Balzacq, à paraître). La dé-sécuritisation - devenue pratiquement un champ d'études en soi au fil des années (Aradau, 2004 ; Floyd, 2010 ; Hansen, 2012 ; Huysmans, 1998) - renvoie à l'idée qu'une question soit extirpée de la sphère sécuritaire et (re)définie comme une question politique. La résilience, qui tient ses origines en psychologie (principalement dans la littérature francophone) et en écologie (surtout dans la littérature anglophone), offre également quelques pistes de réflexion dans le présent contexte, particulièrement lorsqu'elle est conçue comme un continuum de trois types : la résilience comme le maintien du statu quo, la résilience en tant qu'ajustement marginal, et la résilience moteur d'un renouvellement (Bourbeau, 2013b et à paraître). Une société, craignant que l'arrivée de migrants en grand nombre bouscule drastiquement les fondements de son identité collective, pourrait en effet privilégier la résilience comme le maintien du statu quo et ainsi accepter largement les tentatives de sécuritisation proposées par certains agents. La survie de l'objet de référence (l'identité collective) serait ainsi protégée par une stratégie de résilience (visant le maintien du statu quo) face à des chocs exogènes (arrivées réelles ou potentielles de migration massive).

\section{Références bibliographiques}

Abrahamsen Rita and Williams Michael C. (2011) Security Beyond the State: Private Security in International Politics, Cambridge, Cambridge University Press, $272 \mathrm{p}$.

Adamson Fiona (2006) Crossing Borders: International Migration and National Security, International Security, 31 (1), pp. 165-199.

Agier Michel (2002) Aux bords du monde, les réfugiés, Paris, Flammarion, 186 p.

Andreas Peter (2000) Border Games: Policing the U.S.-Mexico divide, Ithaca, Cornell University Press, $158 \mathrm{p}$.

Aradau Claudia (2004) Security and the democratic scene: desecuritization and emancipation, Journal of International Relations and Development, 7 (4), pp. 388-413.

Arendt Hannah (1961) Condition de I'homme moderne, Paris, Calmann-Lévy (traduit de l'américain par Georges Fradier), 328 p.

Auditor General of Canada (2008) Report of the Auditor General of Canada to the House of Commons. Chapter 7: Detention and Removal of Individuals, Ottawa, Canada Border Services Agency.

Austin John (1991) Quand dire c'est faire, Paris, Seuil, 202 p.

Axworthy Lloyd (2000) Notes for an Address by the Honourable Lloyd Axworthy, Minister of Foreign Affairs, to the Middlebury College 200th Anniversary Symposium on International Affairs, Ottawa, Department of Foreign Affairs, March 30.

Axworthy Lloyd (1999) Notes for an Address by the Honourable Lloyd Axworthy, Minister of Foreign Affairs, to the Atlantic Diplomatic Forum, Ottawa, Department of Foreign Affairs, November 5. 
Badie Bertrand and Wihtol de Wenden Catherine (1994) Le defi migratoire : questions de relations internationales, Paris, Presses de la Fondation nationale des sciences politiques, $185 \mathrm{p}$.

Balzacq Thierry (Ed.) (à paraître) Contesting Security. London, Routledge.

Balzacq Thierry (Ed.) (2011) Securitization Theory. How Security Problems Emerge and Dissolve, London, Routledge, 258 p.

Balzacq Thierry (2005) The Three Faces of Securitization: Political Agency, Audience and Context, European Journal of International Relations, 11 (2), pp. 171-201.

Barutciski Michael (2001) Le militantisme juridique et le néo-naturalisme face au droit international positif : les flux massifs de réfugiés et la sécurité nationale, Revue québécoise de droit international, 14 (1), pp. 37-54.

Berthomière William et Hily Marie-Antoinette (2006) Décrire les migrations internationales : les expériences de la co-présence, Revue Européenne des Migrations Internationales, 22 (2), pp. 67-82.

Bigo Didier (2005) La mondialisation de l'insécurité ? Réflexions sur le champ des professionnels de la gestion des inquiétudes et analytique de la transnationalisation des processus d'insécurisation, Cultures \& Conflits, 58, pp. 53-100.

Bigo Didier (1998a) L'immigration à la croisée des chemins, Revue Européenne des Migrations Internationales, 14 (1), pp. 25-46.

Bigo Didier (1998b) Sécurité et immigration : vers une gouvernementalité par I'inquiétude, Cultures \& Conflits, 31-32, pp. 13-38.

Bigo Didier et Guild Elspeth (2003) La mise à l'écart des étrangers : le visa Schengen, Cultures et Conflits, 49-50, pp. 1-135.

Bigo Didier and Tsoukala Anastassia (2008) Understanding (in)security, in Didier Bigo and Anastassia Tsoukala Eds., Terror, Insecurity, and Liberty, London, Routledge, pp. 1-10.

Bloemraad Irene (2006) Becoming a citizen: incorporating immigrants and refugees in the United States and Canada, Berkeley, University of California Press, 202 p.

Bourbeau Philippe (à paraître) Resiliencism and Security Studies: Initiating a dialogue, in Thierry Balzacq Ed., Contesting Security: Resilience, Resistance, Desecuritization and Emancipation, London, Routledge.

Bourbeau Philippe (2013a) Politisation et sécuritisation des migrations internationales: Une relation à définir, Critique Internationale, 61 (4), pp. 127-145.

Bourbeau Philippe (2013b) Resiliencism: Premises and promises in securitization research, Resilience: International Policies, Practices and Discourses, 1 (1), pp. 4-17.

Bourbeau Philippe (2011) The Securitization of Migration. A study of movement and order, London, Routledge, $192 \mathrm{p}$.

Bourbeau Philippe (2002) La Chine et la diaspora chinoise. L'Extrême-Orient russe convoité, Paris, L'Harmattan, $150 \mathrm{p}$.

Bourbeau Philippe (2001) La diaspora chinoise repensée, Études Internationales, 32 (4), pp. 745-767. 
Bourdieu Pierre (1982) Ce que parler veut dire : I'économie des échanges linguistiques, Paris, Fayard, 243 p.

Bradimore Ashley and Bauder Harald (2011) Mystery Ships and Risky Boat People: Tamil Refugee Migration in the Newsprint Media, Metropolis British Columbia Working Paper Series, 11 (02), 46 p.

Brubaker Rogers (1992) Citizenship and nationhood in France and Germany, Cambridge, Mass., Harvard University Press, 270 p.

Butler Judith (1999) Performativity's Social Magic, in Richard Shusterman Ed., Bourdieu. A criticial reader, London, Blackwell, pp. 113-128.

Butler Judith (1997) Excitable Speech: A politics of the performative, London, Routledge, $185 \mathrm{p}$.

Buzan Barry and Wæver Ole (2003) Regions and powers: the structure of international security, Cambridge, Cambridge University Press, 564 p.

Buzan Barry, Wæever Ole and Wilde Jaap de (1998) Security: a new framework for analysis, Boulder, Colo., Lynne Rienner Publishers, 239 p.

Caloz-Tschopp Marie-Claire (2004) Les étrangers aux frontières de l'Europe et le spectre des camps, Paris, La Dispute, $252 \mathrm{p}$.

Campbell Kim (1993) Notes for a Statement by Prime Minister Kim Campbell, Ottawa, Office of the Prime Minister, June 25.

Cannon Lawrence (2010) Discours du ministre Cannon à l'École Munk des affaires internationales de l'Université de Toronto, Ottawa, Ministère des Affaires étrangères et du commerce internationale, 18 juin.

Caplan Elinor (2001a) Notes for an Address by the Honourable Elinor Caplan, Minister of Citizenship and Immigration, to the Canadian Arab Federation, Ottawa, Department of Citizenship and Immigration, November 9.

Caplan Elinor (2001b) Notes for remarks by the Honourable Elinor Caplan, Minister of Citizenship and Immigration, to the Senate Standing Committee on Social Affairs, Science and Technology, Ottawa, Department of Citizenship and Immigration, October 4.

Caplan Elinor (2000a) Notes for an Address by the Honourable Elinor Caplan, Minister of Citizenship and Immigration, to the Canada China Business Council, Ottawa, Department of Citizenship and Immigration, April 25.

Caplan Elinor (2000b) Notes for an Address by the Honourable Elinor Caplan, Minister of Citizenship and Immigration, to the European Union Seminar on Illegal Migration, Ottawa, Department of Citizenship and Immigration, July 20.

Caplan Elinor (2000c) Notes for an Address by the Honourable Elinor Caplan, Minister of Citizenship and Immigration, to the Fourth National Metropolis Conference, Ottawa, Department of Citizenship and Immigration, March 25.

Caplan Elinor (2000d) Notes for an Address by the Honourable Elinor Caplan, Minister of Citizenship and Immigration, to the Maytree Foundation Trends in Global Migration Forum, Ottawa, Department of Citizenship and Immigration, January 12.

Caplan Elinor (1999a) Notes for an Address by the Honourable Elinor Caplan, Minister of Citizenship and Immigration, to the Annual Meeting of the Canadian Council for Refugees, Ottawa, Department of Citizenship and Immigration, December 3. 
Caplan Elinor (1999b) Remarks by the Honourable Elinor Caplan, Minister of Citizenship and Immigration, to the Canadian Club, Ottawa, Department of Citizenship and Immigration, September 9.

Caplan Elinor (1999c) Statement by the Honourable Elinor Caplan, Minister of Citizenship and Immigration, on Illegal Human Smuggling to Canada, Ottawa, Department of Citizenship and Immigration, August 11.

Ceyhan Ayse and Tsoukala Anastassia (2002) The securitization of migration in Western societies: Ambivalent discourses and policies, Alternatives, 27 (Supplement S.), pp. 21-39.

Chrétien Jean (2001) Address by Prime Minister Jean Chrétien on the occasion of a Special House of Commons Debate in response to the terrorist attacks in the United States on September 11, 2001, Ottawa, Office of the Prime Minister, September 17.

CIC (1996) Citizenship and Immigration Detention Policy, Ottawa, Citizenship and Immigration Canada.

CICI (2012) Rapport au Parlement. Les chiffres de la politique de l'immigration et de l'intégration. Année 2011, Paris, Secrétariat général du Comité interministériel de contrôle de l'immigration, République française.

Clochard Olivier (2010) Le contrôle des flux migratoires aux frontières de I'Union européenne s'oriente vers une disposition de plus en plus réticulaire, Carnets de géographes, 1, $18 \mathrm{p}$.

Coderre Denis (2003a) Notes for an Address "Document Integrity and Biometrics: Exploring the Options for our future" by the Honourable Denis Coderre, Minister of Citizenship and Immigration, Ottawa, Department of Citizenship and Immigration, September 19.

Coderre Denis (2003b) Notes for an Address by the Honourable Denis Coderre, Minister of Citizenship and Immigration, to a Forum on Biometrics: Applications and Implications for $\mathrm{ClC}$, Ottawa, Department of Citizenship and Immigration, October 8.

Coderre Denis (2003c) Notes for an Address by the Honourable Denis Coderre, Minister of Citizenship and Immigration, to the Meeting of the Canadian Bar Association, Ottawa, Department of Citizenship and Immigration, May 3.

Coderre Denis (2003d) Notes for an Appearance by the Honourable Denis Coderre, Minister of Citizenship and Immigration, before the Standing Committee on Citizenship and Immigration, Ottawa, Department of Citizenship and Immigration, March 20.

Collectif (2012) Centre et locaux de rétention administrative. Rapport 2011, Paris, Assfam, Forum réfugiés, France terre d'asile, La Cimade, Ordre de Malte France, $292 \mathrm{p}$.

Connelly Matthew and Kennedy Paul (1994) Must It Be the Rest Against the West?, The Atlantic Monthly, December, pp. 61-91.

Cornelius Wayne A., Martin Philip and Hollifield James F. (Eds.) (2004) Controlling immigration: A global perspective, Stanford, Calif., Stanford University Press, $534 \mathrm{p}$.

Crépeau François and Nakache Delphine (2006) Controlling Irregular Migration in Canada. Reconciling Security Concerns with Human Rights Protection, Choices. Institute for Research on Public Policy, 12 (1), pp. 1-42. 
Curley Melissa and Wong Siu-lun (Eds.) (2008) Security and Migration in Asia: the dynamics of securitisation, London, Routledge, $258 \mathrm{p}$.

Dench Janet and Crépeau François (2003) Introduction. Interdiction at the expense of Human Rights: A Long-Term Containment Strategy, Refuge Canada's Periodical on Refugees, 21 (4), pp. 2-5.

Doty Roxanne Lynn (1998) Immigration and the politics of security, Security Studies, 8 (2), pp. 71-93.

Floyd Rita (2010) Security and the Environment: Securitisation theory and US environmental security policy, Cambridge, Cambridge University Press, 230 p.

Freedman Jane (2004) Immigration and insecurity in France, Aldershot, Ashgate, $182 \mathrm{p}$.

Geddes Andrew (2012) Immigration et multiculturalisme en Grande-Bretagne : vers une nouvelle nation civique ?, Politique étrangère, 2, pp. 295-308.

Guild Elspeth and Van Selm Joanne (Eds.) (2005) International Migration and Security: Immigrants as an asset or a threat?, New York, Routledge, 286 p.

Guiraudon Virginie (2010) Les effets de I'Européanisation des politiques d'immigration et d'asile, Politique Européenne, 31 (2), pp. 7-32.

Guiraudon Virginie (2000) Les Politiques d'Immigration en Europe. Allemagne, France, Pays-Bas, Paris, L'Harmattan, 281 p.

Guiraudon Virginie and Joppke Christian (2001) Controlling a new migration world, London ; New York, Routledge, 256 p.

Hansen Lene (2012) Reconstructing desecuritisation: the normative-political in the Copenhagen School and directions for how to apply it, Review of International Studies, 38 (6), pp. 525-546.

Helly Denise (2009) La légitimité en panne?, Cultures \& Conflits, 74 (2), pp. 11-62.

Hollifield James F. (2004) France: Republicanism and the Limits of Immigration Control, in Wayne A.Cornelius, Philip Martin and James F. Hollifield Eds., Controlling Immigration: A global perspective, Stanford, Calif., Stanford University Press, pp. 183-214.

Hollifield James F. (2000) The Politics of International Migration: How can we 'bring the state back in'?, in Caroline B. Brettell and James F. Hollifield Eds., Migration Theory: Talking across disciplines, New York, Routledge.

Huntington Samuel P. (2004) The Hispanic Challenge, Foreign Policy, MarchApril, pp. 1-14.

Huysmans Jef (2006) The Politics of Insecurity. Fear, Migration and Asylum in the $E U$, London, Routledge, $191 \mathrm{p}$.

Huysmans Jef (1998) Dire et écrire la sécurité : le dilemme normatif des études de sécurité, Cultures \& Conflits, 31-32, pp. 177-204.

Joppke Christian and Morawska Ewa T. (Eds.) (2003) Toward assimilation and citizenship: immigrants in liberal nation-states, New York, Palgrave Macmillan, 243 p.

Kaplan Robert (1994) The Coming Anarchy, The Atlantic Monthly, February, pp. 44-76. 
Kenney Jason (2011) Notes pour une allocution prononcée par l'honorable Jason Kenney, C.P., ministre de la Citoyenneté, de I'Immigration et du Multiculturalisme prend la parole à la faculté de droit de I'University of Western Ontario, Ottawa, Ministère de la Citoyenneté, de I'Immigration et du Multiculturalisme, 11 février.

Kenney Jason (2009) Notes pour une allocution prononcée par l'honorable Jason Kenney, C.P., ministre de la Citoyenneté, de I'Immigration et du Multiculturalisme à l'occasion d'un déjeuner-table ronde avec la Chambre de commerce indocanadienne, Ottawa, Ministère de la Citoyenneté, de I'Immigration et du Multiculturalisme, 13 janvier.

Kenney Jason (2008) Notes pour une allocution prononcée par l'honorable Jason Kenney, C.P., député, ministre de la Citoyenneté, de l'Immigration et du Multiculturalisme à I'occasion du 11e Forum diplomatique annuel, Ottawa, Ministère de la Citoyenneté et de I'Immigration, 17 décembre.

Kobelinsky Carolina and Makaremi Chowra (2008) Confinement des étrangers : entre circulation et enfermement, Cultures \& Conflits, 71, pp. 7-11.

La Cimade (2012) Migrations. État des lieux, Paris, La Cimade.

Le Bail Hélène (2008) Nouvelle immigration chinoise et société d'accueil japonaise : entre représentations criminalisantes et ascension sociale, Revue Européenne des Migrations Internationales, 24 (3), pp. 53-77.

Loch Dietmar et Barou Jacques (2012) Les migrants dans I'espace transnational: permanence et changement, Revue Européenne des Migrations Internationales, 28 (1), pp. 7-12.

Loescher Gil (1992) Refugee Movements and International Security, Adelphi Paper 268, London, International Institute for Strategic Studies, 83 p.

Manley John (2000) Notes for an Address by the Honourable John Manley, Minister of Foreign Affairs, to the Third Annual Diplomatic Forum on the Subject of Canada's Foreign Policy Agenda and Priorities, Ottawa, Department of Foreign Affairs, October 20.

Martiniello Marco et Simon Patrick (2005) Les enjeux de la catégorisation. Rapports de domination et luttes autour de la représentation dans les sociétés post-migratoires, Revue Européenne des Migrations Internationales, 21 (2), pp. 7-18.

McDougall Barbara (1992a) Notes for an Address by the Honourable Barbara McDougall, Minister of Foreign Affairs, to the Council of Ministers of the Conference on Security and Cooperation in Europe, Ottawa, Department of Foreign Affairs, January 30.

McDougall Barbara (1992b) Notes for an Address by the Honourable Barbara McDougall, Minister of Foreign Affairs, to the official opening of the Canadian Foreign Service Institute, Ottawa, Department of Foreign Affairs, October 1.

McDougall Barbara (1991) Notes for an Address by the Honourable Barbara McDougall, Minister of Foreign Affairs, to the conference commemorating the 60th anniversary of the Statute of Westminster, Ottawa, Department of Foreign Affairs, December 10.

Miller Mark J. (1998) International Migration and Global Security, in Nana Poku and David T. Graham Eds., Redefining Security: Population movements and national security, London, Praeger, pp. 15-28. 
Mountz Alison (2010) Seeking asylum: human smuggling and bureaucracy at the border, Minneapolis, University of Minnesota Press, 209 p.

Mountz Alison (2004) Embodying the nation-state: Canada's response to human smuggling, Political Geography, 23 (3), pp. 323-345.

Newman Edward and Van Selm Joanne (2003) Refugees and Forced Displacement: International security, human vulnerability, and the state, Tokyo, United Nations University Press, 391 p.

Noiriel Gerard (2008) L'Immigration : naissance d'un " problème " (1881-1883), Agone, 40, pp. 15-40.

OIM (2011) État de la migration dans le monde 2011, Genève, Organisation internationale pour les migrations, $192 \mathrm{p}$.

Oxman-Martinez Jacqueline, Hanley Jill and Gomez Fanny (2005) Canadian Policy on Human Trafficking: A Four-year Analysis, International Migration, 43 (4), pp. 7-29.

Pellerin Hélène and Gabriel Christina (Eds.) (2008) Governing International Labour Migration. Current Isues, Challenges and Dilemmas, London, Routledge, $272 \mathrm{p}$.

Potvin Maryse (2008) Crise des accommodements raisonnables. Une fiction médiatique?, Montréal, Athéna Éditions, 277 p.

Pratt Anna (2005) Securing Borders: Detention and Deportation in Canada, Vancouver, University of British Columbia Press, 290 p.

Rea Andrea et Tripier Maryse (2008) Sociologie de l'immigration. Nouvelle édition, Paris, La Découverte, $120 \mathrm{p}$.

Rodier Claire (2012) Xénophobie business. À quoi servent les contrôles migratoires ?, Paris, La Découverte, $194 \mathrm{p}$.

Rodier Claire (2006) Analyse de la dimension externe des politiques d'asile et d'immigration de I'UE. Synthèse et recommandations pour le Parlement européen, Bruxelles, Parlement Européen, Direction générale pour les politiques externes de I'Union.

Rudolph Christopher (2006) National Security and Immigration: Policy Development in the United States and Western Europe since 1945, Stanford, Stanford University Press, 268 p.

Shain Yossi and Barth Barth Aharon (2003) Diaspora and International Relations Theory, International Organization, 57 (3), pp. 449-479.

Solberg Monte (2006a) Discours de I'honorable Monte Solberg, ministre de la Citoyenneté et de I'Immigration, devant l'Alberta Association of Municipal Districts and Counties, Congrès de l'automne 2006, Ottawa, Ministère de la Citoyenneté et de l'Immigration, 15 novembre.

Solberg Monte (2006b) Discours de l'Honorable Monte Solberg, ministre de la Citoyenneté et de l'Immigration, lors d'une réunion du Comité permanent de la citoyenneté et de l'immigration, Ottawa, Ministère de la Citoyenneté et de I'Immigration, 10 mai.

Tirman John (Ed.) (2004). The Maze of Fear: Security and Migration after 9/11, New York, New Press, 322 p. 
Tribalat Michèle (2012) Immigration et démographie en France, Outre-Terre, 33-34 (3-4), pp. 137-150.

Tsoukala Anastasia (2010) Hooliganisme en Europe. Sécurité et libertés publiques, Montréal, Athéna, $240 \mathrm{p}$.

US Department of Justice (2000) Federal Law Enforcement Officers 2000, Washington, U.S. Department of Justice, Bureau of Justice Statistics.

Van Munster Rens (2009) Securitizing Migration. The politics of risk in the EU, Basingstoke, Palgrave, $177 \mathrm{p}$.

Wæver Ole (1995) Securitization and Desecuritization, in Ronnie D. Lipschultz Ed., On Security, New York, Columbia University Press, pp 46-86.

Weiner Myron (1995) The global migration crisis: challenge to states and to human rights, New York, HarperCollins College Publishers, xiv, 253 p.

Weiner Myron (1993) International Migration and Security, Boulder, Colo., Westview Press, 333 p.

Wimmer Andreas and Glick Schiller Nina (2003) Methodological Nationalism, the Social Sciences, and the Study of Migration: An essay in historical epistemology, International Migration Review, 37 (3), pp. 576-610.

Winter Elke (2011) Us, Them, and Others: Pluralism and National Identity in Diverse Societies, Toronto, University of Toronto Press, 288 p.

Wihtol de Wenden Catherine (2010) La question migratoire au XXle siècle, Paris, Presses de Science Po, 264 p. 


\section{Philippe Bourbeau}

\section{... Processus et acteurs d'une vision sécuritaire des migrations : le cas du Canada}

La perception des migrations internationales a significativement changé depuis la fin de la guerre froide. Auparavant largement traitées sous un angle économique et démographique, les migrations internationales sont depuis quelques années une préoccupation de sécurité de la majorité des pays membres de I'OCDE. Pour décrire ce virage sécuritaire, les chercheurs parlent de la sécuritisation des migrations ou de migrations qui évoluent dans un cadre sécuritaire. Or, si la littérature théorique sur le processus de sécuritisation est bien développée, la base empirique demeure incomplète et limitée, particulièrement l'analyse de contenu de discours réalisée de façon systématique et sur une période de temps relativement longue. Deux objectifs guident cet article. D'une part, l'auteur cherche à analyser, sur la base d'une étude comparative des discours de plusieurs agents politiques au Canada entre 1989 et 2011, le rôle de nombreux agents dans le processus de sécuritisation des migrations. D'autre part, il tente de dégager, en conclusion et sur la base de son analyse, des hypothèses de travail pour les études sur la sécuritisation des migrations.

\section{Process and Agents of the Securitization of Migration: The Case of Canada}

Abrasive rhetoric about international migration is gaining popularity. Nation-states around the globe, especially Western ones, are cracking down on migration for security reasons. Scholars have referred to this current state of affairs as securitized migration or as the securitization of migration. Theoretical studies about the securitization process abound in specialized journals. Yet, empirical studies remain incomplete and limited, particularly systematic content analysis of speeches over a relatively long period of time. The objectives of the article are twofold. First, the article seeks to provide, on the basis of a comparative analysis of several securitizing agents' speeches in Canada between 1989 and 2011, a better understanding of the patterns of engagement of these agents with the phenomenon of securitized migration. Second, the article wants to suggest broader conclusions and avenues for future research worth emphasizing.

\section{$\cdots$ Proceso y actores de una visión de seguridad de las migraciones: el caso de Canadá}

La percepción de las migraciones internacionales ha cambiado de manera significativa desde el final de la guerra fría. Anteriormente estudiadas desde un punto de vista económico y demográfico, las migraciones internacionales constituyen desde hace algunos años una preocupación de seguridad de la mayoría de los países miembros de la OCDE. Para describir este viraje relacionado con la seguridad, los investigadores hablan de la securitización de las migraciones o de migraciones que evolucionan en un marco de seguridad. Existen numerosas publicaciones que explican desde un punto de vista teórico el proceso de securitización. Sin embargo, la base empírica de este proceso sigue siendo incompleta y limitada, particularmente el análisis de contenido del discurso, el cual ha sido realizado de manera sistemática y en un periodo de tiempo relativamente largo. Dos objetivos guían este artículo. En primer lugar, el autor intenta analizar sobre la base de un estudio comparativo de los discursos de varios actores políticos en Canadá entre 1989 y 2011, el rol de varios agentes en el proceso de securitización de las migraciones. En segundo lugar, trata de identificar hipótesis de trabajo, basadas sobre el análisis de sus resultados, que ayuden a entender mejor el proceso de securitización de las migraciones. 